Voix et Images

\title{
Qu'est-ce qu'une femme en noir, un projet de film et des portraits pornographiques ont en commun?
}

\section{Pierre Hébert}

Volume 17, numéro 2 (50), hiver 1992

L’âge de la critique, 1920-1940

URI : https://id.erudit.org/iderudit/200966ar

DOI : https://doi.org/10.7202/200966ar

Aller au sommaire du numéro

Éditeur(s)

Université du Québec à Montréal

ISSN

0318-9201 (imprimé)

1705-933X (numérique)

Découvrir la revue

\section{Citer cet article}

Hébert, P. (1992). Qu'est-ce qu'une femme en noir, un projet de film et des portraits pornographiques ont en commun? Voix et Images, 17(2), 324-332. https://doi.org/10.7202/200966ar d'utilisation que vous pouvez consulter en ligne. 
Roman

\section{Qu'est-ce qu'une femme en noir, un projet de film et des portraits pornographiques ont en commun?}

Pierre Hébert, Université de Sherbrooke

Dire "Je " ne va pas toujours de soi: Jovette Bernier et Jean-Charles Harvey l'ont àppris à leurs dépens. L'affirmation de soi s'est posée de diverses manières dans le roman québécois, et je tiens pour essentielle cette courte phrase ouvrant Les Demi-Civilisés: "Je me nomme Max Hubert." Prononçant pour premier mot "Je", le personnage se plaçait au centre de l'univers, position peu usuelle en cette année 1934. Cela méritait bien condamnation! 
Il revenait cependant au roman des années quarante et cinquante, c'est-à-dire au roman psychologique ou du "cas de conscience ", de porter à un degré plus haut la recherche du moi. Des récits comme Au delà des visages ou Poussière sur la ville sont en effet construits autour d'une problématique, meurtre et adultère en l'occurrence, qui oblige les protagonistés à définir leurs valeurs en accord ou en opposition avec la collectivité qui les entoure. De tels romans $\backslash$ ne sont évidemment possibles que lorsque la société qu'ils présentent ne se fonde plus sur l'unanimité du groupe. Meurtre et adultère auraient été cinquante ans plus tôt des gestes de réprobation; voilà que, polysémiques, ils sont devenus des occasions de questionnement.

Comment le roman des années soixante et soixante-dix pose-t-il la recherche de soi? La problématique nationaliste aurait-elle eu pour effet de ramener l'intérêt vers le collectif? Alors pourquoi tant de romans à la première personne? Relisons par exemple Salut Galarneau! ou encore L'Amélanchier, qui s'ouvre exactement comme Les Demicivilisés: "Je me nomme Tinamer de Portanqueu ....

Mais la recherche de soi se pose d'une tout autre manière dans un certain nombre d'œuvres récentes. Elle ne se fait plus par rapport aux normes collectives ou à une quête sociale. Tout au contraire: nombreux sont les personnages qui, pour (re)trouver leur moi accomplissent un parcours qui ne se pose pas par opposition, mais par décrochage. On ne compte plus les personnages en état de crise: ils prennent conscience que, à quelque part, ils ont dévié de leur idéal et qu'il se sont confinés à répondre aux attentes du social. Ils ont bien joué leur rôle, ils ont bien fonctionné; mais leur moi *véritable ", celui qu'ils ont abandonné sur le bord du chemin, finit par les rattraper. La crise s'instaure: au delà des masques, des rôles, le personnage doit s'arrêter et se demander, sans mensonge: «Que m'est-il arrivé? Qui suis-je vraiment? Où est ma réalité?• Ouvrez presque n'importe quel roman récent (bien sûr, j'exagère... mais si peu!). Vous y lirez, souvent dès le début, quelque chose comme: «Il se sentait désabusé après avoir milité pour de nombreuses causés ${ }^{1}$. Ou encore: "Il approchait de la trentaine quand tout ou à peu près se mit à aller de travers ${ }^{2}$; "J'ai vu passer toutes les modes [...]. Aucune ne m'a vraiment atteint ${ }^{3}$. $\mathrm{Ne}$ manquons pas de noter qu'au départ de ce parcours qui conduit au désabusement, il y a aussi ceux et celles que la masquarade sociale n'intéresse pas: Hamelin, Trudel, Harvey, Mistral. Le premier groupe veut quitter un certain espace social; le second erre...

Les trois romans dont il sera question ici posent d'entrée de jeu cet état de crise. Le premier cas, Deux Semaines en septembre d'André 
Girard, s'amorce par le départ de l'épouse: pour le personnage, c'est l'occasion d'un retour sur soi et d'une interrogation sur les choix qui ont entraîné un reniement des valeurs authentiques. Dans La Danse éternelle de Roger Fournier, le succès d'un cinéaste indique la fin de la masquarade: Jean-Pierre L'Heureux se lance alors dans une entreprise qui l'oppose aux membres de sa société, mais qui représente pour lui un accomplissement essentiel. Enfin, La Jeune Femme et la pornographie de Roger Des Roches pousse à sa limite l'accomplissement de soi dans la gratuité: car ici c'est l'imminence de la mort qui conduit une jeune fille à vivre intensément sa réalité, celle de son corps.

“[...] je rêvais de partir en bateau et je ne suis jamais monté dans la moindre petite chaloupe ${ }^{4}$."

C'est donc un roman des espoirs déçus que nous propose ici André, Girard. Deux Semaines en septembre, c'est tout ce qu'il faut au personnage principal, Clément DaSylva, pour faire le point sur l'éclatement de ses rêves, sur la désagrégation de sa vie de tous les jours. Mais ces deux semaines serviront aussi à lui indiquer par où se trouve le chemin de la vraie vie.

Encore une fois, répétons-le, voici un héros aux abords de la quarantaine qui voit sa vie se défaire. Encore une fois, oui, : car l'on ne compte plus les romans où les personnages, les hommes en l'occurrence, ont la quarantaine difficile. Et pour tous, le même constat s'avère: à quelque part, les projets, les idéals de jeunesse ont fait fausse route. Parfois, le personnage en perdition a la chance de s'en apercevoir tout seul; en d'autres occasions, il lui faut un message brutal qui signale la fin de l'inconscience.

Clémént DaSylva a compris, selon la méthode dure, que tout venait d'être chambardé: au moment où le récit commence, son épouse Hélène l'a quitté depuis une semaine, "comme ça, sans avertissement. (p. 12). Regardons toutefois la situation selon un autre angle: cette rupture oblige DaSylva à chercher où, dans sa vie antérieure, s'est produite l'erreur d'aiguillage. 'Sans doute la meilleure façon de revenir sur son passé est-elle d'y retourner physiquement: ces deux semaines en septembre, Clément DaSylva les passera donc dans son petit village du Saguenay. Et qu'y cherche-t-on d'abord, si ce ne sont ses anciens amis?

DaSylva renoue ainsi avec Réjean Griffin, son camarade de classe du secondaire devenu maintenant sculpteur de totems et de menhirs. En outre, il fera dans son patelin une autre rencontre fortuite, celle d'une étrange femme en noir dont il cherchera à découvrir l'identité. 
Ces deux nouveaux personnages joueront un rôle déterminant dans la quête de Clément DaSylva.

D'abord Réjean Griffin: celui-ci représente l'homme qui a fait de sa vie ce qu'il voulait, quelle qu'ait été la marginalité de ses' choix. Cette découverte, dix ans plus tard, ébranle DaSylva:

Moi qui avais considéré à une certaine époque que le salut de l'homme passait par la métropole, je prenais conscience de la distance qui me séparait de Réjean. [...] D'une façon presque indécente, Réjean me renvoyait l'image d'un homme en pleine possession de ses moyens alors que je m'interrogeais sur le sens de mon aventure audiovisuelle, obsédé par cette femme en fuite avec notre enfant. (p. 41)

En effet, Réjean Griffin s'est donné une vie solide, dans tous les sens du mot. L'aspect massif de ses sculptures envoûte DaSylva et lui procure un sentiment de bien-être qu'il n'avait pas prévu : réconfort, équilibre, lucidité même sont ce qu'il retrouve à la fréquentation de Réjean et de ses œuvres. "Sur cette table de granite, tout devenait clair." (p. 45) Réjean sera ainsi ce premier adjuvant qui permettra au héros de s'avancer plus avant en lui-même, témoignage vivant de valeurs vécues sans compromission et de choix faits en réponse à un élan intérieur plutôt que sous la dictée de la vie sociale. Clément DaSylva sentait confusément, après le départ d'Hélène, qu'il avait écarté une part essentielle de lui-même: "Voilà, me disais-je [...], tu as passé la vingtaine et une bonne partie de la trentaine à ne faire que le faire et, finalement, tu as complètement oublié ta fonction première, celle de voir, de sentir, de regarder:" (p. 15) C'est une nouvelle réalité qui s'ouvre pour DaSylva.

La "femme en noir", dont il découvrira la véritable identité, jouera également un rôle déterminant. Dans une vie qui avait admirablement bien réussi à balàyer les rêves sous le tapis du prévisible, du faire et du confort banal, cette femme en noir insinue le mystère, l'inconnu. DaSylva le comprend bien: "Elle devenait mon saut dans le vide, mon mur à franchir.. (p. 69) Notre personnage, dont la vie est en train de muer, se retrouve ainsi devant une alternative cruciale: ou bien il renonce à aller jusqu'au bout dans son enquête sur cette femme en noir, préférant alors rester dans les limites du prévisible; ou bien il s'enfonce dans son aventure, se démontrant à lui-même qu'il peut "retrouver une part de légèreté qui avait animé [sa] vingtaine" (p. 69).

Comme la fin du roman est à cet égard révélatrice! Pour la première fois, la femme en noir amorce un échange possible avec DaSylva; celui-ci se voit alors aux prises avec la décision de sa vie: "[...]. aller brancher le frigo ou poursuivre le dialogue" (p. 156). 
Brancher le frigo? Image de la vie banale s'il en est une, et qui nous renvoie au début du récit quand DaSylva, justement, avant son départ, avait arraché presque de rage le cordon de l'appareil. Parions 'qu'il optera pour le dialogue. Tant pis pour le frigo.

Ce roman d'André Girard intéresse à plus d'un titre. Très bien écrit, conduit d'une main assurée, raconté sans bavure, sans verbiage, le récit tient constamment l'intérêt à partir d'éléments qui pourtant n'ont rien d'exceptionnels. Mäis l'ensemble est fort bien amené, en particulier par la superposition successive des intrigues: pourquoi Hélène a-t-elle quitté DaSylva? quel est le sens de la quête artistique de Réjean Griffin? qui est la femme en noir? Et; plus que tout, que se passera-t-il de vraiment important durant ces adeux semaines en septembre"?

Une dernière réflexion, enfin, sur Clément DaSylva comme héros de notre temps. Ce héros a trente-cinq, quarante ans. Assez souvent, il a réussi ou, à tout le moins, il s'est construit une petite vie confortable. Mais voilà qu'un jour, pour lui, tout s'écroule parce que sommeillait une réserve qu'il n'avait pas épuisée et qui s'éveille lorsque le prétexte en signale le moment. Ce surplus de vie qui dort en lui, c'est le rêve, le risque, l'imprévu, la vie autre que celle du parcours social objectif signifié par l'épaisseur du curriculum vitæe. L'occasion de là révélation, c'est dans ce roman le départ d'Hélène; l'occasion de la réalisation, ć'est la femme en noir. Dans l'être humain se tapit toujours une volonté d'être, et d'être le plus totalement possible.

Mais la plénitude de l'être peut aussi prendre des directions imprévies; c'est, justement, ce que nous montre maintenant, de Roger Fournier, le second roman.

" [...] il venait de sentir, dans sa chair, au plus profond de son êtré, une espèce de fissure, de lézarde purulente ${ }^{5}$."

La Danse êternelle de Roger Fournier est, dans un certain sens, l'examen de conscience d'un personnage. Mais, curieusement, ce retour sur soi est provoqué par le succès. En effet, le cinéaste JeanPierre L'Heureux vient de mériter tous les éloges pour son dernier film. Et pourtant, le soir de la première, il a écláté en sanglots sanis trop comprendre pourquoi. Bien sûr, ses proches attribuent cette réaction à l'émotion intense du moment; à l'accomplissement artistique exceptionnel du cinéaste. Il n'en est 'rien, car c'est à partir de ce succès que Jean-Pierre L'Heureux amorce une descente en lui-même qui le conduit à vouloir réaliser un projet de film eśsentiel pour lui, mais dont personné ne veut: cette idée d'un film à deux personnages 
et de leur traversée symbolique sur l'eau n'est pas rentable commercialement, lui rétorque-t-on. Malgré tous les refus, L'Heureux persistera dans son ambition personnelle.

Ce parcours narratif en cáche un autre, autrement plus important: Jean-Pierre L'Heureux quitte le monde du réel pour accéder à celui du vrai. Le réel l'a comblé, l'ajpothéose se trouvant dans ce succès qui ouvre le roman. Mais L'Heureux ne découvre-t-il pas, en revanche, que "la vie nous détruit en noús comblant. (p. 55)? Le succès représente justement - ô paradoxe! - la fissure par où se glisse le vrai dans le réel. Notre personnage, encore ici, éprouve le sentiment d'une perte, d'une déviation de sa course authentique: il n'est jamais trop tard pour tourner dos à l'existence imposée par les autres et pour remettre le cap sur le vrai, sur le continent personnel:

Et l'érotisme dans tout cela? Car il s'agit bien, n'est-ce pas, de Roger Fournier..: Oui, il en est question; ou plutôt; l'érotisme se trouve mis en question. Car comment se posent les gestes de l'amour dans cet antagonisme entre le réel et le vrai?

L'érotisme cache mal une forme de désespoir: la passsion qui l'anime est soumise au temps et à la distance des corps. Les amants qui s'ébattent, en réalité se débattent contre la racine du mal, croyant mettre à mort "l'animal invisible qu'ils pourchass[entl toús les deux" (p. 37). Presque rendu au terme de sa course intérieure, Jean-Pierre L'Heureux savourera "le désespoir qu'il y a dans les gestes de l'amour.... (p. 148)

Mais n'est-il pas vrai que ales chants les plus désespérés sont les chants les plus beaux", comme l'a écrit Musset? Que si le réel impose une utilisation normalisée du corps, en revanche, le vrai peut exiger d'y recourir, "littéralement et dans tous les sens"? Si cé corps est la seule façon de mesurer la distance infinie qui nous sépare de l'autre, c'ést aussi le seul moyen; fûtt-il déséspéré, de partager avec l'autre un langage.commun. Le rapport physique avec l'autre cache et montre à la fois le fait que nous sommes seuls.

"La pornographie, c'est lé lieu enfantin où tout dit "oui "...6"

Aussi bien le dire tout de suite, La Jeune Femme et la pornographie est un texte qui m'a considérablement troublé ou, pour dire cela plus positivement, qui m'a mis en mouvement vers des lieux de réflexion où, rarement me semble-t-il, un texte ose nous porter.

Que feriez-vous si l'on vou's annonçait qu'il ne vous reste que quelques mois à vivre? Prendre congé de tout afin d'être plus 
disponible à la vie? Voyager? Continuer votre vie telle que vous la vivez maintenant?

Pour Hélène B., 26 ans, sur qui un verdict fatal a été posé, la décision est claire: elle se suicidera. Mais avant - et c'est là tout le roman - elle décide de se livrer, tout entière et à jamais, comme elle le dit elle-même, à un homme qu'elle ne connaît pas, Robert Y., qu'elle a simplement filé jusque chez lui. Elle se livrera par une série de portraits "pornographiques" qu'elle fera d'elle-même. Qu'elle les lui envoie ou non n'a aucune importance ici: ce qu'il faut retenir, c'est ce mouvement vers l'autre par une série de gestes qui dépassent la camisole de force du quotidien. La Jeune Femme et la pornographie nous présente ces portraits en même temps que la réflexion d'Hélène $B$. révélée, entre autres, par son journal intime.

Qu'est-ce qui troublera tant dans ce roman? Pour certains, on peut supposer que ce seront les descriptions pornographiques elles-mêmes, prises au premier niveau comme exacerbation de la libido (!); pour d'autres, le fait que cela "ne se fait pas ", qu'à tout dire ou tout montrer on paralyse les désirs ou le rêve. Chacun a droit à ses "troubles"... Le mien réside dans l'inversion que fait le roman de Roger Des Roches: la pornographie est un hommage à la vie en même temps qu'une lutte contre la mort. La pornographie, c'est le plus grand maître de vérité qui soit, le chant le plus désespéré mais àussi le plus religieux qui puisse nous unir à cette vie.

Mais comme cela ne tombe pas sous le sens! Pour bien le comprendre, il faut vraiment se mettre dans la peau, dans le corps de cette jeune femme qui va mourir, et qui cherche comment, pour parler comme Georges Bataille, abolir la discontinuité entre les êtres. Tout ce qu'elle a, c'est ce corps qui l'abandonne, et dont elle veut épeler la réalité totale. Elle se photographie dans toutes les positions possibles, afin de n'avoir aucun secret pour cet homme; par la pornographie, elle veut "faire reculer l'échéance de la mort" (p. 96).

La pornographie revendique ainsi un amour intense de la vérité. Le corps est tout à la fois ce qui me circonscrit comme individu et ce qui me permet d'aller à l'autre: Hélène $\mathrm{B}$. a bien compris que c'est en étalant tous les replis de ce corps qu'elle peut surmonter l'isolement: "La pornographie, [...] lorsqu'on la pratique, permet de greffer à son corps les corps des autres, à sa vie, la vie des autres [...].»(p. 98)

Mais, rétorquera-t-on, l'érotisme n'eût-il pas suffi? Absolument pas: "Contrairement à l'érotisme, la pornographie consiste à raconter une histoire, toute l'histoire, au complet, généreusement. ( (p. 42) L'érotisme vit du désir qui lui, est irréalité: il se détruit en s'incarnant. Entre 
l'érotisme et la pornographie, il y a une différence de nature, et non de degré: l'érotisme vit dans la pénombre, il dissimule, il évoque, il dit à demi-mots. La pornographie s'accomplit sous une "lumière royale * (p. 19), elle montre tout, n'ayant pour seul maître que la réalité. Au faire croire de l'érotisme, la pornographie substitue le faire tout court: "On doit toujours contempler un cul en pleine lumière." (p. 127)

Dans La Jeune Femme et la pornograpbie, c'est le corps qui revendique, par la mise à nu, l'abolition de cette ridicule frontière entre l'autre et moi. Il n'y parviendra pas, il n'y parviendra jamais, mais le cri de ses sens ne se taira pas pour autant:

Adulte, on met une frontière, de la tête aux pieds, à ce qu'est notre corps, ceci incluant le dos qu'on ne voit jamais, incluant les zones grises et les points aveugles. L'autre corps, peu importe combien collé, combien intime en paroles et en gestes il est, quelque [sic] soit la pénétration, il reste qu'il ne fait pas partie de soi. Si jamais vous aviez l'intention de me le demander, voilà peut-être le grand thème de toute mon : cuvre: l'autre, c'est l'autre; je veux moi, je veux l'autre?.

La vie est toujours un Dead end. Mais comment expliquer que le thème de la mort, de même que celui de l'érotisme ou de la pornographie hante les lettres québécoises, par les temps qui courent? Il n'y a pas là qu'effets de mode ou pure coïncidence. Remarquons les gens à un salon funéraire, c'est-à-dire ceux quii souffrent vraiment: la mort les porte à se toucher, ce qu'il ne feraient pas, bien sûr, en temps "normal . Face à la mort, le corps s'ex-prime, va en dehors de luimême et cherche l'autre. Le corps d'Hélène B. lutte, lui aussi, contre l'irréductible séparation des êtres, en posant des gestes qui ne'visent aucune négociation ou échange: "Mais pourtant, maintenant que je n'ai plus rien à offrir, voilà que je veux tout donner gratuitement.» (p. 66)

Dans ces trois romans se produit une conversion, dans le premier sens du mot. En effet, chacun des trois personnages se tourne vers soi, se con-vertit donc, la faute ayant été de s'être détourné de soi. Dans ces textes, le "soi * est de rigueur. Je ne dis pas le "moi : cela ferait trop égocentrique... Je dis plutôt le "sọi", à la manière de Fernand Ouellette dans son journal intime. Cependant, cette quête n'isole pas, car dans les trois cas, elle conduit à l'autre dans une relation encore plus authentique. 
Ne réduisons pas ces œuvres à l'expression de l'individualisme contemporain ou au déclin, voire à la disparition des idéologies de rassemblement. Elles nous portent bien au delà de la réduction de l'univers à sphère individuelle. Ces nouveaux romans du "cas de conscience. ne cherchent plus à montrer les conflits des normes sociales, mais plutôt à libérer de celles-ci le parcours unique et authentique de chacun.

Le discours de ces textes est ponctué par une crise, puis une quête: la crise, c'est la rupture d'avec la femme aimée, le succès artistique, un verdict de mort. Quant à la quête, elle est déclenchée par une femme en noir, un projet de film marginal ou une série de portraits pornographiques. La diversité de ces moyens ne doit pas faire écran à ce qui les unit : la réalisation totale et sans compromis de soi, le triomphe de l'idéal personnel sur les exigences sociales.

1. Jean-François Chassay, Obsèques, Montréal, Leméac, 1991, p. 11.

2. André Major, L'Hiver au coeur, Montréal, XYZ, coll. - Novella ,, 1987, p. 11.

3. Jean-Robert Sansfaçon, L'Eau dans l'encrier, Montréal, Quinze, 1989, p. 7.

4. André Girard, Dewx Semaines en septembre, Montréal, Quinze, 1991, 156 p.; voir p. 138.

5. Roger Fournier, La Danse éternelle, Laval, Trois, 1991, 185 p.; voir p. 17.

6. Roger Des Roches, La Jeune Femme et la pornographie, Montréal, Les Herbes rouges, 1991, 132 p.; voir p. 120.

7. André Gervais, Comment découper le corps certain et s'en sortir avec passion. Entretien avec Roger Des Roches;, Voix \& Images, vol. XIII, n॰ 2 (38), hiver 1988, p. 228. 